\title{
A Significant Increase of Estimated Glomerular Filtration Rate After Switching From Fenofibrate to Pemafibrate in Type 2 Diabetic Patients
}

\author{
${\text { Hidekatsu Yanai }{ }^{\mathrm{a}, \mathrm{b}} \text {, Hisayuki Katsuyama }}^{\mathrm{a}}$, Mariko Hakoshima ${ }^{\mathrm{a}}$
}

\begin{abstract}
Background: Dyslipidemia is one of the major risk factors for cardiovascular disease (CVD), along with hypertension, diabetes, smoking and obesity. Approximately $70 \%$ of CVD risk remains even after treatment of elevated low-density lipoprotein-cholesterol (LDL-C) by statins. High triglyceride (TG) and low high-density lipoprotein-cholesterol (HDL-C) level are potential therapeutic targets to prevent CVD. Fibrates were associated with a greater reduction in TG, and a greater increase in HDL-C. Fibrates activate specific transcription factors belonging to the nuclear hormone receptor superfamily, termed peroxisome proliferator-activated receptors (PPARs). Fibrates improve atherogenic dyslipidemia by mediating PPAR $\alpha$. Pemafibrate is a novel member of the selective PPAR $\alpha$ modulator (SPPARM $\alpha$ ) family that was designed to have a higher PPAR $\alpha$ agonistic activity and selectivity than previous fibrates. Here, we aimed to study the influences of the switching from fenofibrate to pemafibrate on metabolic parameters in type 2 diabetic patients.
\end{abstract}

Methods: We retrospectively picked up type 2 diabetic patients who had undergone the switching from fenofibrate to pemafibrate, and compared metabolic parameters before the switching with the data at 3, 6 and 12 months after the switching.

Results: We found 15 patients with type 2 diabetes. Serum alanine aminotransferase significantly decreased at 6 months after the switching as compared with baseline. The estimated glomerular filtration rate (eGFR) significantly increased at 3, 6 and 12 months after the switching from fenofibrate to pemafibrate as compared with baseline. Serum uric acid (UA) levels significantly increased at 3 and 6 months after the switching as compared with baseline. We did not observe changes in other metabolic parameters after the switching.

Manuscript submitted November 2, 2021, accepted November 16, 2021

Published online November 29, 2021

aDepartment of Diabetes, Endocrinology and Metabolism, National Center for Global Health and Medicine Kohnodai Hospital, Chiba, Japan

${ }^{\mathrm{b}}$ Corresponding Author: Hidekatsu Yanai, Department of Diabetes, Endocrinology and Metabolism, National Center for Global Health and Medicine Kohnodai Hospital, 1-7-1 Kohnodai, Ichikawa, Chiba 272-8516, Japan. Email: dyanai@hospk.ncgm.go.jp

doi: https://doi.org/10.14740/cr1333
Conclusion: We observed a significant increase of eGFR and serum UA after the switching from fenofibrate to pemafibrate in type 2 diabetic patients. Recent evidences suggest that the improvement of eGFR is beneficially associated with the development of CVD in type 2 diabetic patients. Considering the impact on eGFR, pemafibrate may effectively reduce CVD as compared with fenofibrate.

Keywords: Cardiovascular disease; Estimated glomerular filtration rate; Fenofibrate; Pemafibrate; Uric acid

\section{Introduction}

Dyslipidemia is one of the major risk factors for atherosclerotic cardiovascular disease (ASCVD), along with hypertension, diabetes, smoking and obesity. The meta-analysis showed that statins reduce the risk of ASCVD by approximately 20-30\%, suggesting that approximately $70 \%$ of ASCVD risk remains even after treatment of high low-density lipoprotein-cholesterol (LDL-C) by statins [1, 2]. High levels of triglyceride (TG)-rich lipoproteins such as intermediate-density lipoprotein (IDL) and very-low-density lipoprotein (VLDL) and low high-density lipoprotein cholesterol (HDL-C) level are commonly observed in patients with type 2 diabetes and obesity [3, 4]. Such atherogenic dyslipidemias are potential therapeutic targets to prevent ASCVD.

An optimal reduction of cardiovascular risk through the management of atherogenic dyslipidemias depends on efficacy of lipid-modulating agents beyond statin-based reduction of LDL-C. The most important class of medications to manage atherogenic dyslipidemias can be fibrates, because fibrates were associated with a greater reduction in $\mathrm{TG}$, and a greater increase in HDL-C [5]. Fibrates activate specific transcription factors belonging to the nuclear hormone receptor superfamily, termed peroxisome proliferator-activated receptors (PPARs) [6]. Three isoforms are identified: PPAR $\alpha, \operatorname{PPAR} \gamma$ and PPAR $\beta / \delta$ [7]. PPAR $\alpha$ is abundant in energy-demanding tissues, such as the liver, kidney, heart and skeletal muscle; PPAR $\gamma$ is predominantly found in adipose tissue, macrophages and the large intestine, whereas PPAR $\beta / \delta$ is more ubiquitous in distribution $[8,9]$. These PPARs are controlled through their interaction with fatty acids (FAs) and their derivatives and are the pharmacological targets for fibrates (PPAR $\alpha$ ) or the insulin sensitizer thiazolidin- 
ediones (PPAR $\gamma)$. Fibrates elevate HDL-C levels via transcriptional induction of synthesis of HDL apolipoproteins by mediating PPAR $\alpha$. By mediating PPAR $\alpha$, fibrates also reduce serum TG by lowering hepatic apo C-III production and by increasing lipoprotein lipase-mediated lipolysis and FA oxidation.

Pemafibrate is a novel member of the selective PPAR $\alpha$ modulator (SPPARM $\alpha$ ) family that was designed to have a higher PPAR $\alpha$ agonistic activity and selectivity than existing PPAR $\alpha$ agonists (such as fibrates) $[8,10]$. Here, we aimed to study the influences of the switching from fenofibrate to pemafibrate on metabolic parameters in type 2 diabetic patients.

\section{Materials and Methods}

We retrospectively picked up type 2 diabetic patients who had undergone the switching from fenofibrate to pemafibrate, and compared metabolic parameters before the switching with the data at 3, 6 and 12 months after the switching. The study protocol was approved by the Ethics Committee of the National Center for Global Health and Medicine, and the study was performed in accordance with the Declaration of Helsinki. The paired $t$-test was used to statistically analyze comparison in metabolic parameters between before and after the switching.

\section{Results}

We found 15 patients with type 2 diabetes. Baseline characteristics for type 2 diabetic patients who had undergone the switching from fenofibrate to pemafibrate are shown in Table 1 . Ten patients had hypertension, nine patients were overweight, and eight patients had received non-fibrate lipid-lowering drugs.

Clinical and biochemical parameters except for estimated glomerular filtration rate (eGFR) and serum uric acid (UA) of patients before and after the switching are shown in Table 2. Only serum alanine aminotransferase significantly decreased at 6 months after the switching as compared with baseline. Other parameters did not show a significant change after the switching. Changes in eGFR and serum UA are shown in Figure 1. The eGFR significantly increased at 3, 6 and 12 months after the switching from fenofibrate to pemafibrate as compared with baseline. Serum UA levels significantly increased at 3 and 6 months after the switching as compared with baseline.

\section{Discussion}

The lipid profile of patients with type 2 diabetes, obesity and metabolic syndrome is characterized by low HDL-C levels and increased TG [3, 4], which has been defined as atherogenic dyslipidemia. Fibrates can be effectively used to manage such atherogenic dyslipidemias. Randomized control trials showed that gemfibrozil, fenofibrate and bezafibrate were significantly effective to prevent CVD events in patients with atherogenic dyslipidemia [11]. In the Fenofibrate Intervention and Event Lowering in Diabetes (FIELD) study, fenofibrate significantly
Table 1. Characteristics for Type 2 Diabetic Patients Who Had Undergone the Switching From Fenofibrate to Pemafibrate ( $\mathrm{n}$ $=15$

\begin{tabular}{|c|c|}
\hline Characteristics & \\
\hline Clinical characteristics & \\
\hline Age (years) & $55.1 \pm 12.0$ \\
\hline Gender (male/female) & $8 / 7$ \\
\hline Body weight (mean $\pm \mathrm{SD}, \mathrm{kg}$ ) & $75.2 \pm 15.6$ \\
\hline Body mass index (mean $\left.\pm \mathrm{SD}, \mathrm{kg} / \mathrm{m}^{2}\right)$ & $27.5 \pm 4.8$ \\
\hline Systolic blood pressure (mean $\pm \mathrm{SD}, \mathrm{mm} \mathrm{Hg}$ ) & $133.4 \pm 12.7$ \\
\hline Diastolic blood pressure (mean $\pm \mathrm{SD}, \mathrm{mm} \mathrm{Hg}$ ) & $79.4 \pm 11.2$ \\
\hline Treatments for type 2 diabetes & \\
\hline Dipeptidyl peptidase-4 inhibitors (n) & 4 \\
\hline Metformin (n) & 7 \\
\hline Sodium-glucose co-transporter 2 inhibitors (n) & 8 \\
\hline Glucagon-like peptide 1 receptor agonists (n) & 3 \\
\hline Sulfonylurea (n) & 1 \\
\hline Insulin (n) & 2 \\
\hline$\alpha$-glucosidase inhibitors (n) & 1 \\
\hline Treatments for hypertension & \\
\hline Angiotensin receptor blockers (n) & 8 \\
\hline Calcium antagonists (n) & 6 \\
\hline Diuretics (n) & 2 \\
\hline$\beta$-blockers (n) & 1 \\
\hline Mineralocorticoid receptor antagonist (n) & 1 \\
\hline Treatments for dyslipidemia & \\
\hline Statins (n) & 2 \\
\hline Ezetimibe (n) & 6 \\
\hline
\end{tabular}

SD: standard deviation.

reduced CVD events in those with low HDL-C or hypertension [12]. The largest effect of fenofibrate to reduce CVD risk was observed in subjects with marked dyslipidemia ( $\mathrm{TG} \geq 2.3$ $\mathrm{mmol} / \mathrm{L}$ and low HDL-C) in whom a $27 \%$ relative risk reduction (95\% confidence interval: $9-42 ; \mathrm{P}=0.005)$ was observed [12]. Different fibrates may have different spectrum of beneficial effects and adverse effects.

In our study, serum UA significantly increased after the switching from fenofibrate to pemafibrate. Fenofibrate influences on serum UA metabolism [13]. Fenofibrate was reported to decrease serum UA levels by increasing its urinary excretion, most likely through the inhibition of urate transporter 1 (URAT1) by fenofibric acid, its major metabolite [14]. The meta-analyses also demonstrated that fenofibrate significantly reduced serum UA levels $[15,16]$. However, reduction of serum UA by pemafibrate was not reported, suggesting that fenofibrate has URAT1 inhibitory effect; however, pemafibrate may not have such effect. The URAT1 inhibitory effect by pemafibrate should be studied in the future.

In our study, eGFR significantly increased after the 
Table 2. Changes in Metabolic Parameters After Switching From Fenofibrate to Pemabibrate

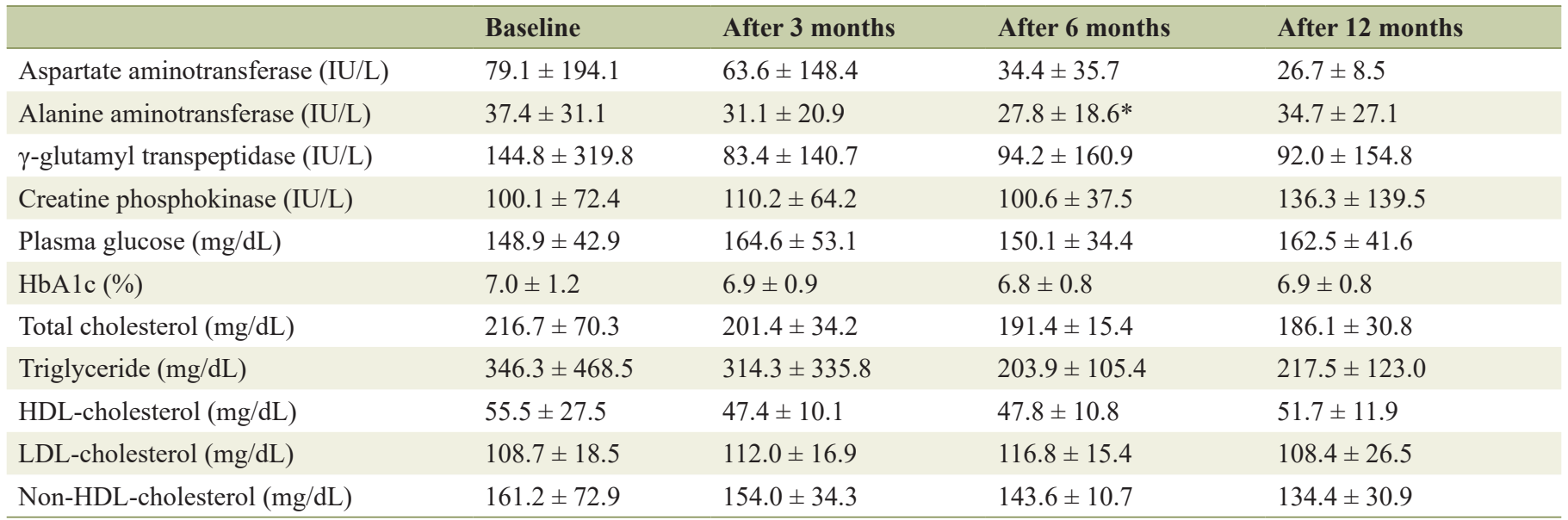

${ }^{*} \mathrm{P}<0.05$ vs. baseline.

switching from fenofibrate to pemafibrate. Fenofibrate was reported to increase serum creatinine levels and decrease the eGFR [17]. In the FIELD Helsinki sub-study, a significant decrease in eGFR was observed in the fenofibrate group [18]. The mechanism of fibrate-induced renal function impairment is unclear. Potential mechanisms include increased muscular production of creatinine, decreased secretion from renal tubules, and a change in the glomerular filtration through altered hemodynamics. Hottelart et al postulated that increased serum creatinine levels result from increased creatinine production [19]. Fibrates reduce the production of vasodilatory prostaglandins, leading to a change in the renal function in patients who experience a rise in the serum creatinine levels [20], which is another possible explanation.

In the multicenter, single-arm, open-label, phase III trial, 0.2 - $0.4 \mathrm{mg} /$ day pemafibrate was administered for 52 weeks to 189 patients with hypertriglyceridemia and an eGFR $\geq 45 \mathrm{~mL} /$

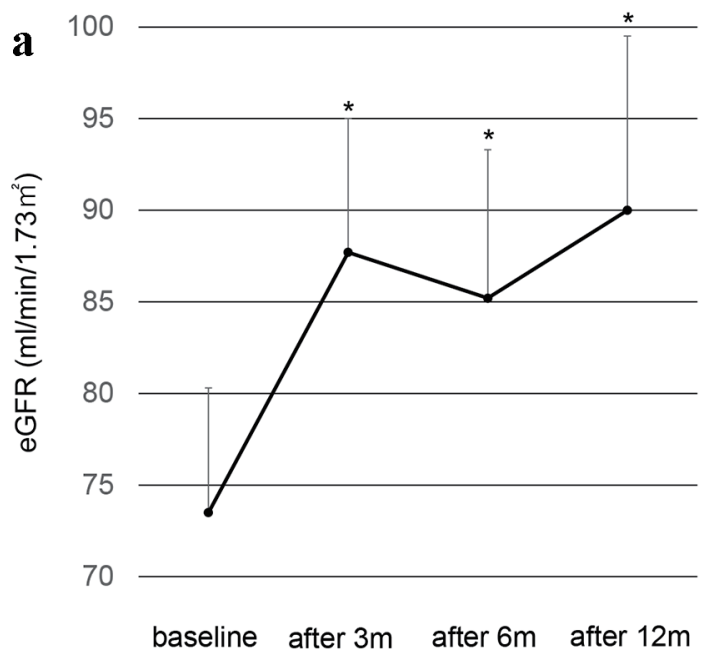

$\min / 1.73 \mathrm{~m}^{2}$ on statin or regardless of eGFR when statin was not administered [21]. There were no significant changes in eGFR over time in any stages of chronic kidney disease (CKD) [21]. Further, pemafibrate showed a good safety profile and efficacy in correcting lipid abnormalities in a broad range of patients, including those with CKD. Fenofibrate is metabolized mainly by the kidneys, whereas pemafibrate is metabolized mainly by the liver [22], which can partially explain a significant increase of eGFR after the switching from fenofibrate to pemafibrate. In an on-drug/off-drug ancillary study to the Action to Control Cardiovascular Risk in Diabetes (ACCORD) Lipid Trial to investigate posttrial changes in serum creatinine, participants with significant initial on-trial increases in serum creatinine $(\geq 20 \%)$ returned to the same level of renal function as participants receiving placebo, suggesting that the fenofibrate-associated on-trial increases in serum creatinine were reversible [23]. A significant increase of eGFR after the

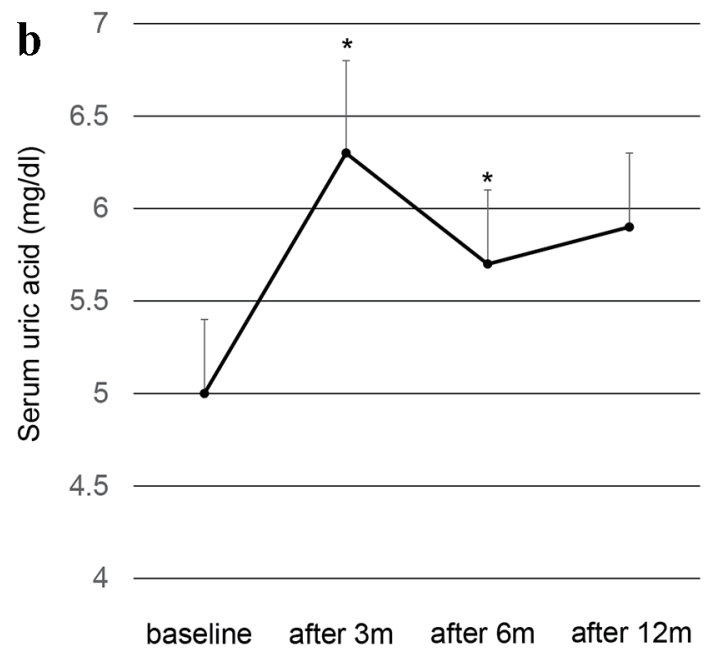

Figure 1. Changes in eGFR (a) and serum uric acid (b) after the switching from fenofibrate to pemafibrate in type 2 diabetic patients. Black circles and error bars indicate mean and SD, respectively. ${ }^{*} \mathrm{P}<0.05$ vs. values at baseline. eGFR: estimated glomerular filtration rate; SD: standard deviation. 
switching from fenofibrate to pemafibrate in our study may reflect the effect of discontinuation of fenofibrate. It remains unknown whether pemafibrate improves eGFR or not in type 2 diabetic patients, which should be studies in the future.

Recent studies using sodium glucose cotransporter 2 inhibitors (SGLT2is) demonstrates that the improvement of eGFR is beneficially associated with the development of CVD in type 2 diabetic patients [24, 25], suggesting a significance of effects of drugs on eGFR when managing cardiovascular risks in patients with type 2 diabetes. Considering the impact on eGFR, pemafibrate may be more beneficially associated with the development of CVD as compared with fenofibrate.

Limitations of the study need to be addressed. This is a cross-sectional study, limiting inferences of causality and its direction. Although treatments for diabetes and hypertension and other lipid-lowering drugs did not change during the study period, we cannot deny that such treatment might influence on the change of eGFR. We should mention further limitations on the small sample size. Further studies, preferably with larger numbers of subjects, will be needed in the future.

In conclusion, we observed a significant increase of eGFR and serum UA after the switching from fenofibrate to pemafibrate in type 2 diabetic patients.

\section{Acknowledgments}

We thank the staffs of the Division of Research Support, National Center for Global Health and Medicine Kohnodai Hospital.

\section{Financial Disclosure}

Authors have no financial disclosures to report.

\section{Conflict of Interest}

The authors declare that they have no conflict of interest concerning this article.

\section{Informed Consent}

Not applicable.

\section{Author Contributions}

HY designed the research, and $\mathrm{MH}$ and $\mathrm{HK}$ collected and analyzed data. HY wrote and approved the final paper.

\section{Data Availability}

The data supporting the findings of this study are available from the corresponding author upon reasonable request.

\section{References}

1. Cholesterol Treatment Trialists' (CTT) Collaboration, Fulcher J, O'Connell R, Voysey M, Emberson J, Blackwell L, Mihaylova B, et al. Efficacy and safety of LDLlowering therapy among men and women: meta-analysis of individual data from 174,000 participants in 27 randomised trials. Lancet. 2015;385(9976):1397-1405.

2. Chapman MJ, Redfern JS, McGovern ME, Giral P. Niacin and fibrates in atherogenic dyslipidemia: pharmacotherapy to reduce cardiovascular risk. Pharmacol Ther. 2010;126(3):314-345.

3. Yanai H, Hirowatari $\mathrm{Y}$, Ito $\mathrm{K}$, Kurosawa H, Tada N, Yoshida H. Understanding of diabetic dyslipidemia by using the anion-exchange high performance liquid chromatography data. J Clin Med Res. 2016;8(5):424-426.

4. Yanai H, Hirowatari Y, Yoshida H. Diabetic dyslipidemia: evaluation and mechanism. Glob Health Med. 2019;1(1):30-35.

5. Abourbih S, Filion KB, Joseph L, Schiffrin EL, Rinfret S, Poirier P, Pilote L, et al. Effect of fibrates on lipid profiles and cardiovascular outcomes: a systematic review. Am J Med. 2009;122(10):962 e961-968.

6. Staels B, Dallongeville J, Auwerx J, Schoonjans K, Leitersdorf E, Fruchart JC. Mechanism of action of fibrates on lipid and lipoprotein metabolism. Circulation. 1998;98(19):2088-2093.

7. Issemann I, Green S. Activation of a member of the steroid hormone receptor superfamily by peroxisome proliferators. Nature. 1990;347(6294):645-650.

8. Fruchart JC. Selective peroxisome proliferator-activated receptor alpha modulators (SPPARMalpha): the next generation of peroxisome proliferator-activated receptor alpha-agonists. Cardiovasc Diabetol. 2013;12:82.

9. Braissant O, Foufelle F, Scotto C, Dauca M, Wahli W. Differential expression of peroxisome proliferator-activated receptors (PPARs): tissue distribution of PPARalpha, -beta, and -gamma in the adult rat. Endocrinology. 1996;137(1):354-366.

10. Yamazaki Y, Abe K, Toma T, Nishikawa M, Ozawa H, Okuda A, Araki T, et al. Design and synthesis of highly potent and selective human peroxisome proliferator-activated receptor alpha agonists. Bioorg Med Chem Lett. 2007;17(16):4689-4693.

11. Tenenbaum A, Fisman EZ. Fibrates are an essential part of modern anti-dyslipidemic arsenal: spotlight on atherogenic dyslipidemia and residual risk reduction. Cardiovasc Diabetol. 2012;11:125.

12. Scott R, O'Brien R, Fulcher G, Pardy C, D'Emden M, Tse D, Taskinen MR, et al. Effects of fenofibrate treatment on cardiovascular disease risk in 9,795 individuals with type 2 diabetes and various components of the metabolic syndrome: the Fenofibrate Intervention and Event Lowering in Diabetes (FIELD) study. Diabetes Care. 2009;32(3):493-498.

13. Yanai H, Adachi H, Hakoshima M, Katsuyama H. Molecular biological and clinical understanding of the pathophysiology and treatments of hyperuricemia and 
its association with metabolic syndrome, cardiovascular diseases and chronic kidney disease. Int J Mol Sci. 2021;22(17):9221.

14. Uetake D, Ohno I, Ichida K, Yamaguchi Y, Saikawa H, Endou H, Hosoya T. Effect of fenofibrate on uric acid metabolism and urate transporter 1. Intern Med. 2010;49(2):89-94.

15. Zhang J, Ji X, Dong Z, Lu J, Zhao Y, Li R, Li C, et al. Impact of fenofibrate therapy on serum uric acid concentrations: a review and meta-analysis. Endocr J. 2021;68(7):829-837.

16. Derosa G, Maffioli P, Sahebkar A. Plasma uric acid concentrations are reduced by fenofibrate: A systematic review and meta-analysis of randomized placebo-controlled trials. Pharmacol Res. 2015;102:63-70.

17. Kim S, Ko K, Park S, Lee DR, Lee J. Effect of Fenofibrate Medication on Renal Function. Korean J Fam Med. 2017;38(4):192-198.

18. Forsblom C, Hiukka A, Leinonen ES, Sundvall J, Groop PH, Taskinen MR. Effects of long-term fenofibrate treatment on markers of renal function in type 2 diabetes: the FIELD Helsinki substudy. Diabetes Care. 2010;33(2):215-220.

19. Hottelart C, El Esper N, Rose F, Achard JM, Fournier A. Fenofibrate increases creatininemia by increasing meta- bolic production of creatinine. Nephron. 2002;92(3):536541.

20. Sica DA. Fibrate therapy and renal function. Curr Atheroscler Rep. 2009;11(5):338-342.

21. Yokote K, Yamashita S, Arai H, Araki E, Suganami H, Ishibashi S, Of The KSGOB. Long-term efficacy and safety of pemafibrate, a novel selective peroxisome proliferator-activated receptor-alpha modulator (SPPARMalpha), in dyslipidemic patients with renal impairment. Int J Mol Sci. 2019;20(3):706.

22. Yamashita S, Masuda D, Matsuzawa Y. Clinical applications of a novel selective PPARalpha modulator, pemafibrate, in dyslipidemia and metabolic diseases. J Atheroscler Thromb. 2019;26(5):389-402.

23. Mychaleckyj JC, Craven T, Nayak U, Buse J, Crouse JR, Elam M, Kirchner K, et al. Reversibility of fenofibrate therapy-induced renal function impairment in ACCORD type 2 diabetic participants. Diabetes Care. 2012;35(5):1008-1014.

24. Yanai H, Hakoshima M, Adachi H, Katsuyama H. Multiorgan protective effects of sodium glucose cotransporter 2 inhibitors. Int J Mol Sci. 2021;22(9):4416.

25. Yanai H. Acute effects of preventing heart failure by sodium-glucose cotransporter 2 inhibitors. Cardiol Res. 2021;12(5):324-326. 